\title{
Risk Factors for Methicillin Resistant Staphylococcus aureus Nasal Colonization of Healthy Children
}

\author{
Babak Soltani ${ }^{1,{ }^{*}}$; Abbas Taghavi Ardakani ${ }^{1}$; Alireza Moravveji ${ }^{2}$; Mahzad Erami ${ }^{3}$; Mostafa \\ Haji Rezaei ${ }^{4}$; Rezvan Moniri ${ }^{3}$; Mansoor Namazi ${ }^{4}$ \\ ${ }^{1}$ Department of Pediatrics, Kashan University of Medical Sciences, Kashan, IR Iran \\ ${ }^{2}$ Department of Community Medicine, Trauma Research Center, Kashan University of Medical Sciences, Kashan, IR Iran \\ ${ }^{3}$ Department of Microbiology, Kashan University of Medical Sciences, Kashan, IR Iran
Trauma Research Center, Kashan University of Medical Sciences, Kashan, IR Iran \\ ${ }^{*}$ Corresponding author: Babak Soltani, Department of Pediatrics, Kashan University of Medical Sciences, Kashan, IR Iran. Tel: + 98-3615575840, Fax: + 98-3615550026, E-mail: babak_ \\ soltani1969@yahoo.com
}

Received: May 06, 2014; Revised: July 02, 2014; Accepted: Jul 25, 2014

\begin{abstract}
Background: Nasal colonization of healthy children with Staphylococcus aureus is an important risk factor for different infections. Detection of colonized individuals with methicillin resistant S. aureus (MRSA) and its eradication is the proper prevention strategy for infection spread in the community and health-care centers.

Objectives: The aim of this study was to determine the prevalence, associated risk factors and antibiotic resistance pattern among healthy children who were nasal carriers of S. aureus.

Patients and Methods: This cross-sectional study was conducted on 350 one month to 14-year-old healthy children living in Kashan/Iran. The nasal specimens were cultured in blood agar medium for S. aureus. Positive cultures were evaluated for cephalothin, co-trimoxazole, clindamycin, ciprofloxacin, oxacillin and vancomycin susceptibility by the disc diffusion method and E-test. Risk factors for nasal carriage of $S$. aureus and MRSA were evaluated.

Results: Frequency of S. aureus nasal carriage was 92 from 350 cases (26.2\%), amongst which 33 (35.9\%) were MRSA. Isolates indicated an overall resistance of $52.2 \%$ to cephalothin, 33.7\% to co-trimoxazol, $26.1 \%$ to ciprofloxacin, $26.1 \%$ to clindamycin, $35.9 \%$ to oxacillin and $4.3 \%$ to vancomycin. Factors associated with MRSA nasal carriage included gender (P value 0.001), age of less than four years (P value 0.016), number of individuals in the family (P value $<0.001$ ), antibiotic use (Pvalue $<0.001$ ) and admission (P value $<0.001$ ) during the previous three months, parental smoking (P value $<0.001)$ and sleeping with parents (P value 0.022).

Conclusions: Age of less than four years, male sex, family size being more than four, antibiotic use and admission during the previous three months, parental smoking and sleeping with parents were independent risk factors for nasal colonization with MRSA.

Keywords:Nasal Colonization; Staphylococcus aureus; MRSA; Risk Factors
\end{abstract}

\section{Background}

Staphylococcus aureus is one of the most common human pathogens that are responsible for a vast spectrum of acute and chronic community and hospital acquired infections $(1,2)$. Anterior nares are the main reservoir of S. aureus in children and adults (3). Asymptomatic colonization is common and $20 \%$ of the healthy population have nasal cavity colonization with $S$. aureus that is a major risk factor for different infections (4). During previous investigations in different communities, the prevalence of nasal carriage of S. aureus in healthy children has been reported as 26.6-52.3\% (5-8). Emergence of resistant S. aureus to current antibiotics and increased prevalence of methicillin resistant $S$. aureus (MRSA) are major obstacles for treatment of infections by this pathogen (9). At first MRSA was reported during the 1960 s, while, currently it is prevalent in health-care centers. In the past, colonization and infections by MRSA were limited to hospitals, but since the 1990s they frequently have been reported in healthy young community members (10-13). Skin and soft tissue infections and severe necrotizing pneumonias are well-known clinical syndromes of MRSA (14). Identification of MRSA nasal carriers and use of control modalities has been recommended for prevention of community and hospital associated infections $(15,16)$.

Considering the substantial prevalence of antibiotic resistance, recognition of antibiotic susceptibility patterns is imperative for decolonization and treatment of $S$. aureus infections. Despite noticeable previous studies regarding the prevalence of nasal carriage of $S$. aureus and MRSA in healthy children, a few investigations have been carried out about their associated risk factors. In this survey, antibiotic susceptibility of isolated nasal S. aureus among healthy children and associated risk factors for nasal MRSA carriage were evaluated.

\section{Objectives}

The goal of this investigation was to determine the

Copyright (C) 2014,Ahvaz Jundishapur University of Medical Sciences; Published by Kowsar. This is an open-access article distributed under the terms of the Creative Commons Attribution-NonCommercial 4.0 International License (http://creativecommons.org/licenses/by-nc/4.0/) which permits copy and redistribute the material just in noncommercial usages, provided the original work is properly cited. 
prevalence, associated risk factors and antibiotic resistance patterns among healthy children who were nasal carriers of MRSA.

\section{Patients and Methods}

\subsection{Study Population}

In this cross-sectional study, 350 one-month to 14-yearold healthy children were evaluated between July 2012 and March 2013. Specimens were collected by cluster random sampling from four health-care centers in Kashan, Iran. The children had referred to the centers for vaccination, growth monitoring or periodic examinations. They were examined by a pediatrician. Children who had acute or chronic respiratory infections, chronic medical disorders, those who needed admission or emergency care, and cases with skin infections were excluded from the study. Informed consent was obtained from parents following explanations about the study. The accompanied parent was interviewed and a questionnaire was filled. The questionnaire contained demographic characteristics and risk factors associated with S. aureus and MRSA nasal carriage such as age, sex, number of individuals in the family, parental smoking, sleeping with parents, antibiotic usage and hospitalization during the past three months. The ethics committee of Kashan University of Medical Sciences approved the study (approval code 823). Sample size was calculated by consideration of the $28.4 \%$ prevalence for $S$. aureus nasal isolation (7), $(\mathrm{d}=0.07, \alpha=$ 0.05 and design effect of 1.5).

\subsection{Nasal Sample Collection and Bacterial Isola- tion}

Sampling was conducted by rotating a moistened sterile cotton swab with sterile saline in vestibule of both anterior nares twice. The collected swabs were inserted in Amies tube transport media with charcoal (HiMedia, Mumbai, India) at a temperature between $2-4^{\circ} \mathrm{C}$ and transported to the microbiology laboratory of Kashan Shahid Beheshti hospital within four hours. Swabs were cultured on mannitol salt agar (MSA) (Merk, Germany) at $35^{\circ} \mathrm{C}$ for 48 hours. Yellowish colonies growing on MSA were subcultured on blood agar (Merk, Germany) for 24 hours. Growing colonies were identified as $S$. aureus by morphology of colony, Gram staining, and catalase, coagulase and DNase production in tube tests $(17,18)$.

\subsection{Antibiotic Susceptibility Pattern}

Screening test for antibiotic resistance was done on positive cultures by the Kirby-Bauer disc diffusion method according to the Clinical and Laboratory Standard Institute (CLSI) (19). The incubated colonies on blood agar were transported by a sterile loop onto Mueller-Hinton agar (Merk, Germany) with the antibiotic containing discs (Mast, UK) placed on top for 24 hours at $35^{\circ} \mathrm{C}$. The discs included: cephalothin $(30 \mu \mathrm{g})$, co-trimoxazole (1.25/23.75 $\mu \mathrm{g})$, clindamycin $(2 \mu \mathrm{g})$, oxacillin $(1 \mu \mathrm{g})$, vancomycin (30 $\mu \mathrm{g})$ and ciprofloxacin $(5 \mu \mathrm{g})$. After an elapse of the mentioned time, the inhibition zones of isolates around the discs were measured and compared with standard guidelines. If the following inhibition zones were observed the sample was considered resistant; cephalothin $\leq 14 \mathrm{~mm}$, co-trimoxazole $\leq 10 \mathrm{~mm}$, clindamycin $\leq 14 \mathrm{~mm}$, oxacillin $\leq 10 \mathrm{~mm}$, vancomycin $\leq 14 \mathrm{~mm}$ and ciprofloxacin $\leq 15 \mathrm{~mm}$ (19). American type culture collection (ATCC) 25923 S. aureus was used as the control isolate for antimicrobial susceptibility detection (19).

Minimal inhibitory concentration (MIC) breakpoints of, cephalothin $\geq 32 \mu \mathrm{g} / \mathrm{mL}$, co-trimoxazole $\geq$ M8/152 $\mu \mathrm{g} / \mathrm{mL}$, clindamycin $\geq 4 \mu \mathrm{g} / \mathrm{mL}$, ciprofloxacin $\geq 4 \mu \mathrm{g} /$ $\mathrm{mL}$, oxacillin $\geq 4 \mu \mathrm{g} / \mathrm{mL}$ and vancomycin $\geq 16 \mu \mathrm{g} / \mathrm{mL}$ were considered resistant (19). MICs were assessed by Etest strips (Liofilchem, Italy). E-test strips were applied to Mueller-Hinton agar plates, which were inoculated with a solution of strains with optical density of 0.5 McFarland standard, using sterile loops. The E-test MIC was measured at the bottom of the inhibition zone intersected by the E-test strip. After screening by the disc diffusion test, resistant strains were confirmed by the E-test. However, the E-test was performed for all S. aureus positive cultures to evaluate oxacillin and vancomycin resistance.

\subsection{Statistical Analysis}

Data analysis was conducted by the SPSS statistical software, version 16. For descriptive results, frequencies and percentages were used. Demographic and associated risk factors for MRSA were analyzed by the Chi-square and Fisher's exact tests, odds ratios and confidence intervals. Data distributions were evaluated using the Kolmogorovsmirnov test. According to the abnormal distribution of age and number of individuals in the family, for their comparison with two independent groups the Mannwhitney U test was used. Some variables were entered in to multivariate logistic regression if bivariate analysis resulted a P value of less than 0.1. All P values were two-sided and those less than 0.05 were considered significant.

\section{Results}

Overall, 350 children with mean age of $7.06 \pm 4.25$ years and age range of one month to 14 years were evaluated. Half of the cases were males with the other half being females. Mean age was $7.29 \pm 4.08$ and $6.84 \pm 4.42$ years among females and males, respectively with no significant difference (P value 0.3). Frequency of $\leq 4,5-9$ and 10- 14 year-old groups was 114 (32.6\%), 119 (34\%) and 117 (33.4\%), respectively.

Among all cases, 92 (26.3\%) had positive nasal cultures for S. aureus, from which 30 (32.6\%) cases were female and $62(67.4 \%)$ male, with this difference being significant (P value $<0.001$ ). Mean age of positive and negative cultures was $6.65 \pm 4.46$ and $7.21 \pm 4.14$ years, respectively 
Soltani B et al.

(P value 0.22). Risk factors associated with nasal S. aureus colonization are shown in Table 1 . From the positive cultures, $26.1 \%$ were sensitive to all antibiotics, $22.8 \%$ were resistant to one, $21.7 \%$ to two, $16.3 \%$ to three, $5.4 \%$ to four, $4.3 \%$ to five and $3.3 \%$ to six antibiotics. The most resistance rate was towards cephalothin (52.2\%) and the least resistance rate was towards vancomycin (4.3\%). Frequency of antibiotic resistance in nasal S. aureus carriers is indicated in Table 2. Prevalence of MRSA among positive cultures was $35.9 \%$. Male gender increased the odds ratio of MRSA carriage fourteen times (P value 0.001). The mean age of MRSA and methicillin sensitive S. aureus (MSSA) carriers didn't differ significantly (P value 0.116 ) yet the age group of $\leq 4$ years was associated with MRSA colonization (P value 0.016).

There was a significant association between antibiotic use and admission during the recent three months ( $P$ value $<0.001$ ), number of individuals in the family ( $P$ value $<0.001)$, sleeping with parents (P value 0.019) and parental smoking (P value $<0.001$ ) with MRSA nasal colonization. Table 3 represents associated risk factors and antibiotic susceptibility of MRSA nasal carriers.

\begin{tabular}{|c|c|c|c|c|c|}
\hline Factors & Positive No. (\%) & Negative No. (\%) & Pvalue & 95\% CI & OR \\
\hline Age group, y & & & 0.026 & - & - \\
\hline$\leq 4$ & $37(40.2)$ & $77(29.8)$ & & & \\
\hline $5-9$ & $21(22.8)$ & $98(38)$ & & & \\
\hline $10-14$ & $34(37)$ & $83(32.2)$ & & & \\
\hline Family size & & & $<0.001$ & $7.09-22.65$ & 12.68 \\
\hline$\leq 4$ & $38(41.3)$ & $232(89.9)$ & & & \\
\hline $4<$ & $54(58.7)$ & $26(10.1)$ & & & \\
\hline Parental smoking & & & $<0.001$ & $11.99-49.64$ & 24.4 \\
\hline Yes & $50(54.3)$ & $12(4.7)$ & & & \\
\hline No & $42(45.7)$ & $246(95.3)$ & & & \\
\hline Sleeping with parents & & & $<0.001$ & $1.82-4.9$ & 2.98 \\
\hline Yes & $58(63)$ & $94(36.4 \%)$ & & & \\
\hline No & $34(37)$ & $164(63.6)$ & & & \\
\hline Admission in the previous three months & & & $<0.001$ & $1.91-5.31$ & 3.18 \\
\hline Yes & $41(44.6)$ & $52(20.2)$ & & & \\
\hline No & $51(55.4)$ & $206(79.8)$ & & & \\
\hline Antibiotic use in the previous months & & & $<0.001$ & $4.47-13.04$ & 7.63 \\
\hline Yes & $53(57.6)$ & $39(15.1)$ & & & \\
\hline No & $39(42.4)$ & $219(84.9)$ & & & \\
\hline Gender & & & $<0.001$ & $1.61-4.37$ & 2.65 \\
\hline Female & $30(32.6)$ & $145(56.2)$ & & & \\
\hline Male & $62(67.4)$ & $113(43.8)$ & & & \\
\hline
\end{tabular}

Table 2 . Frequency of Antibiotic Susceptibility in Nasal Staphylococcus aureus Carriers

\begin{tabular}{lll}
\hline Antibiotics & Sensitive & Resistant \\
\hline Cephalothin & $44(47.8 \%)$ & $48(52.2 \%)$ \\
Co-trimoxazole & $61(66.3 \%)$ & $31(33.7 \%)$ \\
Ciprofloxacin & $68(73.9 \%)$ & $24(26.1 \%)$ \\
Clindamycin & $68(73.9 \%)$ & $24(26.1 \%)$ \\
Oxacillin & $59(64.1 \%)$ & $33(35.9 \%)$ \\
Vancomycin & $88(95.7 \%)$ & $4(4.3 \%)$ \\
\hline
\end{tabular}


Soltani B et al.

\begin{tabular}{|c|c|c|c|c|c|}
\hline \multirow[t]{2}{*}{ Variables } & \multicolumn{2}{|c|}{ Methicillin Resistance } & \multirow[t]{2}{*}{ Pvalue } & \multicolumn{2}{|c|}{ Logistic Regression } \\
\hline & MSSA, No. (\%) & MRSA, No. (\%) & & $\mathrm{OR}(95 \% \mathrm{CI})$ & Pvalue \\
\hline Gender & & & $<0.001$ & $14(3.07-63.91)$ & 0.001 \\
\hline Male & $31(52.5)$ & $31(93.9)$ & & $\leq$ & \\
\hline Female & $28(47.5)$ & $2(6.1)$ & & & \\
\hline Age, $y$, Mean \pm SD & $7.3 \pm 4.42$ & $5.5 \pm 4.66$ & 0.12 & - & - \\
\hline Number of individuals in the family, Mean \pm SD & $4.3 \pm 0.97$ & $5.3 \pm 0.78$ & $<0.001$ & - & - \\
\hline Antibiotic use in the previous three months & & & $<0.001$ & $7.62(2.58-22.5)$ & $<0.001$ \\
\hline Yes & $25(42.4)$ & $28(84.8)$ & & & \\
\hline No & $34(57.6)$ & $5(15.2 \%)$ & & & \\
\hline Admission during the previous three months & & & $<0.001$ & $6.6(2.55-17.05)$ & $<0.001$ \\
\hline Yes & $17(28.8)$ & $24(72.7)$ & & & \\
\hline No & $42(71.2)$ & $9(27.3)$ & & & \\
\hline Family size & & & $<0.001$ & $9.9(3.07-31.64)$ & $<0.001$ \\
\hline$\leq 4$ & $34(57.6)$ & $4(12.1)$ & & & \\
\hline$>4$ & $25(42.4)$ & $29(87.9)$ & & & \\
\hline Age groups (years) & & & 0.016 & - & - \\
\hline$\leq 4$ & $18(30.5)$ & $19(57.6)$ & & & \\
\hline $5-9$ & $18(30.5 \%)$ & $3(9.1)$ & & & \\
\hline $10-14$ & $23(39)$ & $11(33.3)$ & & & \\
\hline Parental smoking & & & $<0.001$ & $7.04(2.52-19.69)$ & $<0.001$ \\
\hline Yes & $23(39)$ & $27(81.8)$ & & & \\
\hline No & $36(61)$ & $6(18.2)$ & & & \\
\hline Sleeping with parents & & & 0.019 & $3.13(1.18-8.34)$ & 0.022 \\
\hline Yes & $32(54.2)$ & $26(78.8)$ & & & \\
\hline No & $27(45.8)$ & $7(21.2)$ & & & \\
\hline Cephalothin resistance & & & 0.003 & $3.89(1.54-9.81)$ & 0.004 \\
\hline Sensitive & $35(59.3)$ & $9(27.3)$ & & & \\
\hline Resistant & $24(40.7)$ & $24(72.7)$ & & & \\
\hline Co-trimoxazole resistance & & & 0.074 & $2.24(0.92-5.48)$ & 0.077 \\
\hline Sensitive & $43(72.9)$ & $18(54.5)$ & & & \\
\hline Resistant & $16(27.1)$ & $15(45.5)$ & & & \\
\hline Ciprofoxacin resistance & & & 0.03 & $2.84(1.09-7.39)$ & 0.033 \\
\hline Sensitive & $48(81.4)$ & $20(60.6)$ & & & \\
\hline Resistant & $11(18.6)$ & $13(39.4)$ & & & \\
\hline Clindamycin resistance & & & 0.002 & $4.63(1.73-12.42)$ & 0.002 \\
\hline Sensitive & $50(84.7)$ & $18(54.5)$ & & & \\
\hline Resistant & $9(15.3)$ & $15(45.5)$ & & & \\
\hline Vancomycin resistance & & & 0.015 & - & - \\
\hline Sensitive & $59(100)$ & $29(87.9)$ & & & \\
\hline Resistant & $0(0)$ & $4(12.1)$ & & & \\
\hline Multi drug resistant & & & $<0.001$ & $8.65(3.13-23.9)$ & $<0.001$ \\
\hline Yes & $8(13.6)$ & $19(57.6)$ & & & \\
\hline No & $51(86.4)$ & $14(42.4)$ & & & \\
\hline
\end{tabular}

${ }^{\mathrm{a}}$ MSSA, methicillin-sensitive Staphylococcus aureus, MRSA, methicillin-resistant Staphylococcus aures, OR, odds ratio, CI, confidence interval. 
Soltani B et al.

\section{Discussion}

According to this study, prevalence of nasal colonization with S. aureus among one-month to fourteen-yearold children was $26.3 \%$. In a survey by Tabbarai et al. (19) on 1193 school-aged children, prevalence of nasal carriage was $16.3 \%$, out of which $34.8 \%$ were MRSA, while peak age of nasal carriage was 6-12 years old and vancomycin resistance rate was $1.7 \%$. Ciftci et al. (7) in Turkey worked on four to six-year-old children and reported $28.4 \%$ for the prevalence of nasal colonization which was consistent with our investigation. In Taiwan, prevalence of nasal colonization among two to 60-month-old children was $23.2 \%$ of which MRSA was present in $7.8 \%$ of cases and peak age of MRSA was two to six months old. Furthermore, day care attendance and family size were risk factors for MRSA nasal carriage (20).

In our study, MRSA carriage was more than that of the Taiwanese report but risk factors were comparable. Other studies have reported the prevalence of nasal S. aureus colonization as $32.1 \%$ in South Korea, $40 \%$ in Tanzania and $18.1 \%$ in USA $(5,6,21)$, which were inconsistent with our study. The different in prevalence of nasal carriage between our study and others may be due to the various age groups studied. For example, two different surveys carried our during 2009 and 2010 in India, reported that nasal carriage among 5-15 and 1-5 year-old children was $52.3 \%$ and $6.3 \%$, respectively $(8,17)$. Furthermore, other characteristics such as socioeconomic status were not determined in these studies.

In the present study, MRSA prevalence among positive nasal cultures was $35.9 \%$, which was compatible with the study by Tabbarai et al. (19) (34.8\%). In other investigations, MRSA prevalence among healthy $S$. aureus nasal carriers varied from $0.3 \%$ (7) to $18.9 \%$ (6) that was significantly lower than our survey. Furthermore, even in societies with prevalence of $40 \%$ and $52.3 \%$ for nasal colonization, MRSA prevalence has been reported as $10.5 \%$ and $3.89 \%$, respectively $(5,8)$. In the present study, the highest susceptibility of MRSA was towards vancomycin and the least sensitivity was towards cephalothin. Huang et al. (20) detected no MRSA resistance to vancomycin and teicoplanin while $99.1 \%$ resistance to penicillin and 9\% sensitivity to clindamycin (54.5\% in our study) was reported. Concordant results have been reported by some researches $(6,17)$ yet MRSA clindamycin susceptibility in the investigation of ko et al. (6) was $61.1 \%$ which was more than our study and other mentioned reports. In this study, prevalence of vancomycin sensitive MRSA was $87.9 \%$, while in other studies this was reported as $100 \%(6,17,20)$.

Comparison of the present research with previous studies indicates more judicious antibiotic prescription is needed for children. Although the vast majority of children with community acquired MRSA nasal colonization are self-limited during a one-year period (22), some of them are at risk of recurrent skin and soft tissue infections thus decolonization of these cases is recommended (23-25). Furthermore, with regards to the high prevalence of nasal carriage of MRSA in our investigation, decolonization of healthy preschool children is prudent.

This study revealed that male gender, antibiotic use and hospitalization during the past three months, number of individuals in the family being more that four, age group of less than four years, parental smoking and sleeping with parent were associated risk factors for MRSA nasal carriage among healthy children. A few researches have been conducted regarding the risk factors of MRSA nasal carriage among healthy children. Fritz et al. $(22,23)$ identified some risk factors for nasal carriage of MRSA in healthy children that included outpatient visit in the past six months, surgery during the previous one year, history of immune deficiency and systemic infections, more than two people per bedroom (crowded home), a household member working at a health care center, a household member aged 19 to 27 years old or more than 60 years old and daycare attendance. Furthermore, the relationship with close contacts such as sharing a bath towel with MRSA nasal carriage was ruled out (26) which appeared incompatible with our findings (close contact via sleeping with parents was associated with MRSA nasal colonization). Some studies have claimed that the household member who works at a hospital is the only risk factor for nasal MRSA carriage (27, 28). Pathak et al. (17) concluded that a large family size was associated with $S$. aureus nasal carriage but recent hospitalization was not. Oppositely, we found that large family size and recent admission were risk factors of carriage.

Our study has some limitations, firstly, it was cross-sectional and didn't differentiate persistent from transient nasal colonization, thus obtaining another nasal culture after one year (cohort design) is recommended to detect persistent carriage over time, which is a major source of community infections. Secondly, it is possible that the isolated strains are not representative of the community and investigations with larger sample sizes are needed in the future. Thirdly, the family socioeconomic status was not evaluated in our research and it would be better to consider this in future studies. Fourthly, we did not confirm MRSA by mecA genes and also didn't find a Vancomycin-resistant S. aureus (VRSA) gene, thus more investigations in this field are imperative. Lastly, the impact of seasonal changes on S. aureus nasal colonization was not evaluated in our study, thus further studies are recommended.

In conclusion, in the present study the prevalence of nasal S. aureus colonization was $26.3 \%$ out of which $35.9 \%$ were MRSA. Furthermore, male sex, antibiotic use and admission in the past three months, crowded family, parental smoking and sleeping with parents were factors associated with MRSA nasal carriage.

\section{Acknowledgements}

The authors of this article are grateful to the microbiology laboratory personnel of Kashan Shahid Beheshti Hospital for collecting the samples and performing the lab 
tests. We would also like to thank the health-care center personnel for their cooperation.

\section{Author's contributions}

Development of the original idea: Babak Soltani. Study concept and design: Babak Soltani and Abbas Taghavi Ardakani. Analysis and interpretation of data: Alireza Moravveji. Data collection: Mostafa Haji Rezaei and Mansoor Namazi. Preparation of the manuscript: Babak Soltani and Mostafa Haji Rezaei. Laboratory testing: Mahzad Erami and Rezvan Moniri. Revision of the manuscript: Babak Soltani.

\section{Funding Support}

Funding support for this study was provided by the Kashan University of Medical Sciences, grant number 91004.

\section{References}

1. von Eiff C, Becker K, Machka K, Stammer H, Peters G. Nasal carriage as a source of Staphylococcus aureus bacteremia. Study Group. NEngl J Med. 2001;344(1):11-6.

2. Wertheim HF, Vos MC, Ott A, van Belkum A, Voss A, Kluytmans JA, et al. Risk and outcome of nosocomial Staphylococcus aureus bacteraemia in nasal carriers versus non-carriers. Lancet. 2004;364(9435):703-5.

3. Wertheim HF, Melles DC, Vos MC, van Leeuwen W, van Belkum A, Verbrugh HA, et al. The role of nasal carriage in Staphylococcus aureus infections. Lancet Infect Dis. 2005;5(12):751-62.

4. Weidenmaier C, Goerke C, Wolz C. Staphylococcus aureus determinants for nasal colonization. Trends Microbiol. 2012;20(5):243-50.

5. Moyo SJ, Aboud S, Blomberg B, Mkopi N, Kasubi M, Manji K, et al. High nasal carriage of methicillin-resistant Staphylococcus aureus among healthy Tanzanian under-5 children. Microb Drug Resist. 2014;20(1):82-8.

6. Ko KS, Lee JY, Baek JY, Peck KR, Rhee JY, Kwon KT, et al. Characterization of Staphylococcus aureus nasal carriage from children attending an outpatient clinic in Seoul, Korea. Microb Drug Resist. 2008;14(1):37-44

7. Ciftci IH, Koken R, Bukulmez A, Ozdemir M, Safak B, Cetinkaya Z. Nasal carriage of Staphylococcus aureus in 4-6 age groups in healthy children in Afyonkarahisar, Turkey. Acta Paediatr 2007;96(7):1043-6

8. Chatterjee SS, Ray P, Aggarwal A, Das A, Sharma M. A communitybased study on nasal carriage of Staphylococcus aureus. Indian J Med Res. 2009;130(6):742-8.

9. Onanuga A, Temedie TC. Nasal carriage of multi-drug resistant Staphylococcus aureus in healthy inhabitants of Amassoma in Niger delta region of Nigeria. Afr Health Sci. 2011;11(2):176-81.

10. Herold BC, Immergluck LC, Maranan MC, Lauderdale DS, Gaskin RE, Boyle-Vavra S, et al. Community-acquired methicillin-resistant Staphylococcus aureus in children with no identified predisposing risk. JAMA. 1998;279(8):593-8.

11. Gosbell IB. Methicillin-resistant Staphylococcus aureus: impact on dermatology practice. Am J Clin Dermatol. 2004;5(4):239-59.

12. Centers for Disease C, Prevention.. Methicillin-resistant Staphylo- coccus aureus skin or soft tissue infections in a state prison--Mississippi, 2000. MMWR Morb Mortal Wkly Rep. 2001;50(42):919-22.

13. Centers for Disease C, Prevention.. Four pediatric deaths from community-acquired methicillin-resistant Staphylococcus aureus - Minnesota and North Dakota, 1997-1999. MMWR Morb Mortal Wkly Rep. 1999;48(32):707-10.

14. Zetola N, Francis JS, Nuermberger EL, Bishai WR. Community-acquired meticillin-resistant Staphylococcus aureus: an emerging threat. Lancet Infect Dis. 2005;5(5):275-86.

15. Muto CA, Jernigan JA, Ostrowsky BE, Richet HM, Jarvis WR, Boyce JM, et al. SHEA guideline for preventing nosocomial transmission of multidrug-resistant strains of Staphylococcus aureus and enterococcus. Infect Control Hosp Epidemiol. 2003;24(5):362-86.

16. Kuehnert MJ, Kruszon-Moran D, Hill HA, McQuillan G, McAllister SK, Fosheim G, et al. Prevalence of Staphylococcus aureus nasal colonization in the United States, 2001-2002. J Infect Dis 2006;193(2):172-9.

17. Pathak A, Marothi Y, Iyer RV, Singh B, Sharma M, Eriksson B, et al Nasal carriage and antimicrobial susceptibility of Staphylococcus aureus in healthy preschool children in Ujjain, India. BMC Pediatr. 2010;10:100.

18. Mohajeri P, Gholamine B, Rezaei M, Khamisabadi Y. Frequency of Mupirocin Resistant Staphylococcus aureus Strains Isolated From Nasal Carriers in Hospital Patients in Kermanshah.J Microbiol.1970;5(4):560-3.

19. Tabbarai A, Ghaemi E, Fazeli MR, Behnampour N. Prevalence of Staphylococci Aureus nasal carrier in healthy school students in Gorgan. Gorgan Univ Medic Sci J. 2001;3(2):6-11.

20. Huang YC, Hwang KP, Chen PY, Chen CJ, Lin TY. Prevalence of methicillin-resistant Staphylococcus aureus nasal colonization among Taiwanese children in 2005 and 2006. J Clin Microbiol. 2007;45(12):3992-5.

21. Miller MB, Weber DJ, Goodrich JS, Popowitch EB, Poe MD, Nyugen V, et al. Prevalence and risk factor analysis for methicillinresistant Staphylococcus aureus nasal colonization in children attending child care centers. J Clin Microbiol. 2011;49(3):1041-7.

22. Fritz SA, Krauss MJ, Epplin EK, Burnham CA, Garbutt J, Dunne WM, et al. The natural history of contemporary Staphylococcus aureus nasal colonization in community children. Pediatr Infect Dis J. 2011;30(4):349-51.

23. Fritz SA, Epplin EK, Garbutt J, Storch GA. Skin infection in chil dren colonized with community-associated methicillin-resistant Staphylococcus aureus. J Infect. 2009;59(6):394-401.

24. Ellis MW, Hospenthal DR, Dooley DP, Gray PJ, Murray CK. Natural history of community-acquired methicillin-resistant Staphylococcus aureus colonization and infection in soldiers. Clin Infect Dis. 2004;39(7):971-9.

25. Creech CB, Beekmann SE, Chen Y, Polgreen PM. Variability among pediatric infectious diseases specialists in the treatment and prevention of methicillin-resistant Staphylococcus aureus skin and soft tissue infections. Pediatr Infect Dis J. 2008;27(3):270-2.

26. Fritz SA, Garbutt J, Elward A, Shannon W, Storch GA. Prevalence of and risk factors for community-acquired methicillin-resistant and methicillin-sensitive staphylococcus aureus colonization in children seen in a practice-based research network. Pediatrics 2008;121(6):1090-8.

27. Nakamura MM, Rohling KL, Shashaty M, Lu H, Tang YW, Edwards KM. Prevalence of methicillin-resistant Staphylococcus aureus nasal carriage in the community pediatric population. Pediatr Infect Dis J. 2002;21(10):917-22.

28. Creech CB, 2nd, Kernodle DS, Alsentzer A, Wilson C, Edwards KM. Increasing rates of nasal carriage of methicillin-resistant Staphylococcus aureus in healthy children. Pediatr Infect Dis J. 2005;24(7):617-21. 